Voix et Images

voixetimages

\title{
La poésie et l'expérience de soi
}

\section{André Brochu}

Volume 26, numéro 1 (76), automne 2000

\section{L'immonde}

URI : https://id.erudit.org/iderudit/201527ar

DOI : https://doi.org/10.7202/201527ar

Aller au sommaire du numéro

\section{Éditeur(s)}

Université du Québec à Montréal

\section{ISSN}

0318-9201 (imprimé)

1705-933X (numérique)

Découvrir la revue

Citer cet article

Brochu, A. (2000). La poésie et l'expérience de soi. Voix et Images, 26(1),

183-186. https://doi.org/10.7202/201527ar d'utilisation que vous pouvez consulter en ligne.

https://apropos.erudit.org/fr/usagers/politique-dutilisation/ 


\title{
La poésie et l'expérience de soi
}

\author{
André Brochu, Université de Montréal
}

On a beau décréter l'indispensable autonomie du texte littéraire par rapport au réel ou au vécu, le langage n'existe jamais seul. Il traîne, charrie de l'expérience humaine, et le poème est souvent l'expression des mouvements vrais de la conscience, de l'inconscient même. L'atlante villemairienne, le sadique selon Roberge, l'amoureuse brisée de Danielle Fournier en témoignent.

D'ambre et d'ombre, de Yolande Villemaire $^{1}$, réunit la plupart de ses recueils depuis le premier, Machinet-elle, daté de 1974, jusqu'aux suites inédites d'aujourd'hui. C'est une somme d'environ deux cents pages, moins considérable qu'on ne l'aurait supposé. Il est vrai que $D u$ côté biéroglyphe de ce qu'on appelle le réel et Adrénaline ${ }^{2}$ en sont absents, le dernier étant de dimensions particulièrement imposantes. Quoi qu'il en soit, le livre donne une bonne idée de la poésie de Villemaire et de son évolution.

Avec une audace appréciable, la poète présente ses textes dans un ordre chronologique inversé, de sorte que sont reproduites en dernier les plaquettes des années soixante-dix (un ultime poème daté de l'an 2000 faisant seul contrepoids à cette production juvénile). On remonte donc vers la voix déjà originale et encore mal placée, mais pleine de fraîcheur, de la jeune femme de vingt-cinq ans, depuis la voix plus sombre mais tou- jours capable d'émerveillement de l'écrivaine actuelle.

Peu de style (hélas?) dans cette poésie qui s'accommode d'une syntaxe simple et ne courtise guère la métaphore. La dimension poétique est constituée par un usage très sûr du vers libre, lequel est manié avec beaucoup de naturel et d'efficacité (c'est là une question de rythme et non, simplement, de métrique, et on connaît le rôle majeur que joue le rythme en poésie $^{3}$ ), et par la succession d'énoncés qui nous font passer d'idées en soi peu surprenantes à d'autres idées capables de créer la nécessaire résonance signifiante. Il ne s'agit pas de poésie intellectuelle (même si l'auteure en aurait les moyens), mais les idées, au sens le plus large du mot, y ont une grande importance car elles sont les dépositaires de la charge poétique. La façon de représenter le réel et de le reconstruire par des références qui l'agrandissent et lui donnent un sens, références notamment à la sagesse indienne et à d'autres antiques civilisations telle l'Égypte, à des pratiques magiques comme le surréalisme (Rrose Sélavy) ou la télépathie, est une stratégie majeure du texte. Elle introduit le lecteur dans un univers à la fois familier et déconcertant, et fait peser un continuel soupçon sur le réel. L'Orient, avec ses épaisseurs de mystère et d'exotisme, de sacré aussi, constitue le principal facteur de poétisation. 
Une autre dimension se dessine pourtant en contrepoint: l'américanité, avec sa culture du cyberespace et sa langue, qui occupe une bonne place dans le poème, se mêlant sans complexe aux vocables français ou franco-québécois (la shed, le mononcle...). Valorisation de l'instant, de la sensation, de l'énergie, des communications nouvelles, d'un futur accueilli avec frénésie: New York, "quartz et mica", est la patrie emblématique de cette hypermodernité. Yolande Villemaire est une fille de ce qu'on a appelé la contre-culture on pourrait encore parler du Nouvel $\hat{A} \mathrm{e}^{4}$. Et elle n'éprouve aucune difficulté à réunir les temps et les espaces les plus éloignés - le plus présent et le très ancien, le voisin immédiat et l'absolument distant, la tradition et la rupture. La suspension des lois du réel permet l'accueil intégral du monde, sans doute sur le mode onirique (ce wishfull thinking qu'on rencontre aussi dans les grands livres calligraphiés de Chamberland), mais c'était un prélude à la mondialisation actuelle, que sert si bien la technologie du virtuel.

Toutefois, malgré l'appel effréné de l'ailleurs, Yolande Villemaire reste fidèle à son passé, personnel et collectif. "Guidée par l'esprit de mes ancêtres amérindiens / Dans cette vallée du Maharastra / Où j’ai planté un arbre pour ma grand-mère / Je danse en sari lilas / Une petite Indienne dans mes bras / Pour bercer ma propre enfance" (p. 125). Plusieurs poèmes, aussi bien récents que plus anciens, nous ramènent avec une naïveté délibérée à l'enfance catholique très fervente, à partir de fois plus exotiques mais aussi ardentes qui sont celles de l'adulte, et la poète tente de les concilier en elle: "J'ai prié la Vierge de m'aider à concilier / La foi de mon enfance / Et les divinités païennes de mes autres vies." (p. 134). L'essentiel est de croire, de donner un sens à sa vie en relation avec les autres et avec le monde, de vivre son existence comme un lieu de découvertes fondé dans le sentiment d'une transcendance (plus féminine, sans doute, que virile, celle d'un Dieu mère ${ }^{5}$ ). L'humour, je l'ai noté jadis à propos d'Adrénaline, empêche l'auteur de sombrer dans le vertige des croyances de pacotille, qui ont tant d'attrait sur les Occidentaux en mal d'oraisons fortes. La poésie de Villemaire garde un côté constamment expérimental - elle tente la fusion de toutes les sagesses, même les plus éloignées de la raison, et même de cette science, de cette technique qui inventent au jour le jour notre avenir, les font avec la problématique abracadabrante des vies antérieures - et fait ainsi une synthèse du refoulé de la conscience occidentale, autour de sa propre conscience de fille sensible, intelligente, vibrante et bien campée dans le théâtre du réel.

Si j'évoque ici le théâtre, c'est que Yolande Villemaire, qui a pratiqué la poésie/performance et qui est une lectrice remarquable de ses textes sur scène, écrit une poésie destinée à l'écoute plus qu'à la froide réception visuelle. L'aplomb de l'énoncé, dominant la terreur charnelle et concrète de "la petite fille" tirée hors de son paradis et menacée par "l'avalanche" des mauvais esprits, partagée entre les sollicitations contradictoires de "l'ambre" et de "l'ombre", tient lieu d'une poétique plus savante qui sous-tendrait un véritable 
travail de l'écriture. À cet égard, la poésie de Villemaire est surtout affaire d'expression et de représentation, et la narration, parfois bien prosaïque, y tient une place considérable, par exemple dans le long poème intitulé "Ganeshpuri, le 5 octobre 1990" (p. 96-109), qui raconte une expérience privilégiée de communication avec une femme Guru. On s'y sent tout près, du reste, de la littérature édifiante, bien connue des catholiques, où le sens critique est totalement recouvert par l'adoration.

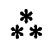

Le crime n'existe pas, proclame, dès le titre, le dernier recueil d'Éric Roberge $^{6}$. Cet enseignement vient de Sade, l'" anti-père " dont un poème liminaire fait l'éloge. Sade affirme que la nature ne peut être outragée, rien ne lui étant extérieur et rien, à l'intérieur, ne lui étant contraire. Il s'ensuit que la possibilité même du crime n'existe pas (p. 7). Roberge en tire une leçon, non pas de libertinage mais de poésie. Autrement dit, par delà les lubriques et souvent meurtrières fantaisies qui l'accompagnent, la pensée sadienne pose un principe aussi précieux que décapant, le matérialisme, qui est une exaltation de la vie. La poésie du poète trifluvien, rien moins que lubrique, tente de dire le monde sans gommer les aspects violents qui le transissent, mais sans céder non plus au vertige de la complaisance.

Le résultat n'est ni tout à fait facile ni tout à fait convaincant. La logique paradoxale faible qui est à l'œuvre ne se laisse pas toujours bien décoder. On relève dans maints poèmes des traces de la carrière militaire $^{7}$ autrefois embrassée par l'écri- vain (et qui n'est pas sans surmotiver la référence à Sade: le soldat n'est-il pas quelqu'un pour qui "le crime n'existe pas", ayant pour métier de l'accomplir, en toute impunité, sans préoccupation morale?). "Notre sauvagerie / est peut-être une falaise / où se jettent notre colère / et nos civilisations / La mousse / la braise arrogante / déposée sur les ruines / et le lierre banalement / la parole des amoureux." (p. 45) Ces quelques vers donnent un assez juste aperçu de la poésie de Roberge, qui formule côte à côte des traits relatifs à la violence du monde et d'autres qui la nient. Ainsi, l'amour est une constante dans des poèmes qui font aussi le procès de l'existence, et cette double veine atteint son apogée dans l'avant-dernière section, "Oubliettes", dont les poèmes sont consacrés à la fille de l'auteur, Juliette (deux ans). Cette fois, le paradoxe devient convaincant car il est surmonté, résolu en de fort belles images pourtant au bord du saugrenu, images d'une grande tendresse:

Quand nous te soulevons
au bout de nos bras
quand nous te faisons danser
ton innocence
est un colis propre
et piégé
qu'on dépose tout doucement
dans le cabinet des apocalypses
(p. 55 )

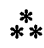

De Danielle Fournier, un livre au titre chargé de connotations on ne peut plus humaines: Ne me dis plus jamais qui je suis ${ }^{8}$. La relation de couple portée à sa conséquence extrême, la rupture, constitue la matière du discours. Ce qui pourrait inspirer 
plus naturellement un roman de type sentimental, voire un téléroman, prend place en poésie, non sans quelque audace, et donne lieu à un soliloque fait de bribes, où le sujet s'entretient le plus souvent lui-même, à la deuxième personne. S'y entremêlent les notations intérieures et les circonstances quotidiennes:

tu aurais tant voulu

qu'il te reste ton désir

rien d'autre

que ce verre plein (p. 77)

Le verre est celui de l'alcool consolateur, et une tragédie bien concrète se trouve ainsi évoquée. Privée de son désir envolé avec l'infidèle, la femme trouve où elle peut le courage de se reconquérir soi-même. Tout cela est expliqué dans le communiqué de presse qui accompagne le recueil et qui en éclaire les arrièreplans. Cette pièce manque-t-elle au lecteur qui doit se satisfaire d'une quatrième de couverture plus laconique? Un poème devrait pouvoir se passer d'explication...

La poésie, qui est suggestion, n'est pas l'absence du référent mais son ouverture à d'autres référents par le travail de l'écriture. Ce travail, du reste, existe dans les poèmes de $\mathrm{Da}$ nielle Fournier, en particulier au début du recueil: “À qui appartiens-tu, trouée et sexe mordu sous le coup des mots sans âge?" (p. 18) La vérité du corps humilié s'y exprime en mots vrais et singuliers. Seulement, il aurait fallu à l'auteure prendre un peu plus de distance par rapport à son sujet, non pas pour convertir son vécu en littérature, mais pour nourrir le projet premier de son écriture des contingences tragiques de la vie.

Pour mieux comprendre ce qu'il en est du discours poétique et de sa capacité de signifier bien au delà de ce qu'il énonce dans l'immédiat du texte, il faut lire le magnifique ouvrage que vient de publier Gilles Marcotte, intitulé Le lecteur de poè$m e{ }^{9}$. À partir de textes québécois, français et même en langue étrangère, il pratique de convaincantes et très fines microanalyses qui débouchent toujours sur une compréhension synthétique du poème ou de l'œuvre. Il y a là une leçon de méthode et, pour tout dire, de litterrature puisque cette dernière peut aussi se retrouver dans le texte critique, quand celui-ci interroge vraiment, comme c'est ici le cas, le souverain langage du poète.

1. Yolande Villemaire, D'ambre et d'ombre, Trois-Rivières/Marseille, Écrits des Forges/L'Orange bleue, 2000, $236 \mathrm{p}$.

2. Yolande Villemaire, $D u$ côté hiéroglyphe de ce qu'on appelle le réel, Montréal, Les Herbes rouges, 1982, 172 p.; Adrénaline, Montréal, Éditions du Noroît, 1982, 76 p. J'ai rendu compte de ces deux livres importants au moment de leur parution dans Voix et Images (vol. VIII, n³, printemps 1983); texte repris dans André Brochu, Tableau du poème. La poésie québécoise des années quatre-vingt, Montréal, XYZ éditeur, p. 184-185.

3. Henri Meschonnic, Critique du rytbme, Paris, Verdier, 1982, $730 \mathrm{p}$.

4. J'utilise ces termes en profane absolu!

5. On connaît l'implication féministe de l'écrivaine.

6. Éric Roberge, Le crime n'existe pas, TroisRivières, Écrits des Forges, 2000, $70 \mathrm{p}$.

7. Par exemple, ces motifs guerriers : - tir nourri ", "douilles vides" (p. 32), "le visage armé... ", "Explosion / Implosion- (p. 34), -À grandes enjambées / cognées du talon / les tambours / et les caisses claires / le chaos battant / étrivières et asphyxie * (p. 35), "la culasse dans l'herbe affaissée. (p. 46). Certains poèmes semblent être des critiques, à la fois énergiques et pas toujours explicites, de la vie militaire. (p. $37,38 \ldots$ )

8. Danielle Fournier, Ne me dis plus jamais qui je suis, Laval, Trois, 2000, $108 \mathrm{p}$.

9. Gilles Marcotte, Le lecteur de poèmes, Montréal, Boréal, 2000, $210 \mathrm{p}$ 